\title{
«Zukunft mit Komplementärmedizin»
}

\author{
Die Bundesversammlung empfiehlt dem Stimmvolk mit 78,5 Prozent, den \\ Verfassungsartikel «Zukunft mit Komplementärmedizin» von Ständerat Rolf \\ Büttiker (FDP SO) anzunehmen. Der Gegenentwurf zur zurückgezogenen Ini- \\ tiative wird Volk und Ständen 2009 unterbreitet werden. Die Union schweize- \\ rischer komplementärmedizinischer Ärzteorganisationen (UNION) hofft, dass \\ die FMH die Position des Parlamentes unterstützt.
}

\section{Jörg Fritschi}

Präsident der Union schweizerischer komplementärmedizinischer Ärzteorganisationen
Korrespondenz:

Dr. med. Jörg Fritschi Im Noll 38

CH-4148 Pfeffingen

dr.fritschi@hin.ch

\begin{abstract}
Verbreitung in der Ärzteschaft
Einige Kollegen sind noch immer der Ansicht, dass Schul- und Komplementärmedizin durch weltanschauliche Gräben unvereinbar getrennt seien. Schul- und ärztliche Komplementärmedizin ergänzen sich in der Praxis aber vorteilhaft. Viele Arztpraxen und Kliniken, welche beide Methodengruppen integriert anwenden, setzen diese Leistungen erfolgreich und kostengünstig ein.

Viele Ärzte haben das Potential der Komplementärmedizin (KM) erkannt. Rund 1700 freipraktizierende Ärztinnen und Ärzte, 23 Prozent aller Grundversorger, sind Mitglieder der UNION. Ein erheblich grösserer Teil, rund 40 Prozent, ist zumindest partiell komplementärmedizinisch tätig. Gemäss einer noch unveröffentlichten Studie der KIKOM ziehen weitere 40 Prozent Kollegen die KM bei Bedarf hinzu; insgesamt setzen damit 80 Prozent aller Hausärzte KM direkt oder indirekt ein.
\end{abstract}

\section{Der Gegenentwurf der Bundesversamm- lung \\ Art. $118 a$ (neu) Komplementärmedizin \\ Bund und Kantone sorgen im Rahmen ihrer Zuständigkeiten für die Berücksichtigung der Komplementärmedizin.}

\section{Die politischen Kernforderungen}

\section{Grundversicherungsleistungen}

Der Gegenvorschlag verlangt explizit keine Änderung der gesetzlichen Vorgaben, sondern einfach den korrekten Vollzug des KVG. Fünf ärztliche Leistungen der KM (anthroposophische Medizin, Homöopathie, Neuraltherapie, Phytotherapie, Traditionelle Chinesische Medizin) müssen aus folgenden Gründen wieder in die Grundversicherung aufgenommen werden:

\section{Krankenversicherungsgesetz}

Der Ausschluss der fünf komplementärmedizinischen Methoden aus der Grundversicherung verstösst gegen geltendes Recht: Der Gesundheitsminister hat sich laut seinen schriftlichen Erklärungen nur auf schulmedizinische Kriterien abgestützt. Gemäss einem Bundesgerichtsentscheid [1] darf sich die Beurteilung aber nicht auf eine rein schulmedizinische Optik beschränken. Zudem haben das Programm Evaluation Komplementärmedizin (PEK) [2] und die einzelnen Forschungsberichte gezeigt, dass die Methoden die gesetzlichen WZW-Vorgaben (WZW = Wirksamkeit, Zweckmässigkeit, Wirtschaftlichkeit) durchaus erfüllen. Der PEK-Bewertungsausschuss hat in der PEK-Draft-Version vom 14. März 2005 empfohlen, die anthroposophische Medizin, die Homöopathie und die Phytotherapie im Leistungskatalog zu belassen. Neuraltherapie und die Traditionelle Chinesische Medizin wurden aus zum Teil formellen Gründen differenzierter beurteilt; der Bewertungsausschuss attestiert aber, dass es sich um wirksame und sinnvolle Therapien handelt. Die detaillierte Konsultation der einzelnen PEK-Studien - mehrere wurden erst nach der Publikation des Schlussberichts publiziert - zeigt, dass der WZW-Nachweis gemäss den für den PEK-Prozess definierten Vorgaben erbracht wurde. Diese Vorgaben («Kriterien Heusser») wurden von der Leistungskommission am 20. März 1998 genehmigt und in das «Handbuch zur Standardisierung der medizinischen und wirtschaftlichen Bewertung medizinischer Leistungen» [3] aufgenommen. Überraschend wurden die positiven Empfehlungen des PEK-Bewertungsausschusses im vier Wochen später publizierten Schlussbericht ersatzlos gestrichen; beibehalten wurden hingegen die insgesamt positiven Bewertungen aller fünf Methoden [4]. Heikel ist der Umstand, dass die politische Entscheidung anscheinend durch eigene, ursprünglich nicht vorgesehene Analysen des Bundesamts für Gesund- 
heit (BAG) inspiriert wurde, deren Daten und Quellen bis heute nicht veröffentlicht wurden.

So hat das internationale PEK-Review-Board am 27. September 2005 in seiner Konsensstellungnahme geschrieben: «Wer den zusammenfassenden Schlussbericht zum PEK-Projekt als richtig beurteilt, wird wohl die politische Entscheidung als inkonsistent mit dem PEK-Ergebnis beurteilen.» [5]

\section{Therapie(wahl)freiheit}

Der Bundesrat schreibt in seiner Botschaft (06.066) zur ursprünglichen Volksinitiative, dass die Therapiewahlfreiheit für Patienten weitgehend erfüllt sei und die Therapiefreiheit der Ärzte nicht eingeschränkt werde. Diese Aussagen entsprechen keineswegs den Tatsachen, denn:

- kranke und ältere Personen können keine Zusatzversicherung für KM abschliessen, weil eine strenge Risikoselektion erfolgt;

- nicht alle Personen können sich eine Zusatzversicherung für KM leisten (oder die Leistungen privat bezahlen).

Gemäss Schätzungen des Bundesrates und der Helsana haben 30 Prozent der Versicherten (2,23 Mio.) keine Zusatzversicherung für KM. Die freie Arztwahl wird für diese Patientengruppe, viele davon mit chronischen Erkrankungen, dauerhaft eingeschränkt.

\section{Verstoss gegen Solidarität in der Grundversicherung}

Gemäss PEK-Schlussbericht gibt es bei bestimmten Kinderkrankheiten, in der Schwangerschaft oder bei psychischen Störungen oft keine Alternativen oder nur solche mit einem höheren Risikopotential. Auch bei 50 Prozent aller Krebspatienten werden komplementäre Therapien angewandt, die jedoch nur teilweise über die Grundversicherung abgerechnet werden können.

\section{Kosteneffizienz}

Die von Ärzten praktizierte KM hat 2004 in der Grundversicherung 25 Millionen Franken gekostet $(0,134$ Prozent [6]); alle konnten sie in Anspruch nehmen. Seit dem Ausschluss betragen die Prämien in der Zusatzversicherung neu mindestens 250 Mio. Franken; die Differenz fliesst grösstenteils den Privatversicherungen zu. PEK hat nachgewiesen, dass die ärztliche KM auch bei Berücksichtigung unterschiedlicher Patientenkollektive v. a. aufgrund preiswerter Arzneimittel gleich teuer oder günstiger als die Schulmedizin zu stehen kommt.

Für die Vergütung durch die Grundversicherung gelten strenge Vorgaben: Für die jeweilige
Disziplin ist der FMH-Fähigkeitsausweis erforderlich (Phytotherapie: Ausweis der UNION); es braucht weder neue Reglemente noch zusätzliche TARMED-Positionen.

Eine Ausweitung des Leistungskatalogs ist weder erforderlich noch absehbar:

- Es gibt keine zusätzlichen von Ärzten praktizierten komplementärmedizinischen Richtungen, die die Voraussetzungen nach Art. 32 KVG erfüllen.

- Nichtärztliche Therapeuten werden weiterhin über die Zusatzversicherungen abrechnen.

\section{Forschung und Lehre}

Die KM ist in Lehre und Forschung vermehrt zu berücksichtigen. Jede Ärztin, jeder Arzt soll in der Ausbildung wenigstens die Grundsätze von Heilmethoden kennenlernen, die von einer Mehrheit der Bevölkerung nachgefragt werden; die Verordnung über die Prüfungen für Ärzte (811.112.2) verlangt denn auch, dass sich die Ärzteausbildung an den Bedürfnissen der Bevölkerung orientiert. Die fünf aufzunehmenden Methoden sollen jede mindestens eine ordentliche Professur erhalten. Für die Richtungen, die mit Arzneimitteln arbeiten, braucht es zusätzlich pharmazeutische Professuren. Gefordert werden insgesamt elf Professuren, d.h. ein Prozent der bestehenden medizinischen und pharmazeutischen Professuren, wovon zwei bereits bestehen. Gemäss einer Schätzung der GDK belaufen sich die Kosten pro Professur auf rund eine Million Franken; die Finanzierung soll durch Drittmittel und Umschichtung von bestehenden Mitteln erreicht werden. Nebst qualifizierten inländischen Bewerbern stehen bei Bedarf Forscher aus ausländischen Universitäten zur Verfügung; die komplementärmedizinische Lehre ist in angloamerikanischen und einigen Ländern der EU weit fortgeschritten.

\section{Arzneimittel}

Das Heilmittelgesetz sieht im Artikel 14 eine vereinfachte Zulassung für Arzneimittel der KM vor. Demgegenüber hat Swissmedic über das Ziel hinausschiessende, bürokratische Zulassungsverordnungen erlassen und verlangt übermässig hohe Gebühren. Als Folge verschwinden unzählige traditionelle Heilmittel vom Markt, obwohl sie auch gemäss Swissmedic - keine nennenswerten Nebenwirkungen verursachen. Dafür blüht der Schwarzmarkt via Internet, der sich jeder Kontrolle entzieht. Das Heilmittelgesetz muss daher vom Parlament zwingend angepasst werden, damit Swissmedic ihren Ermessenspielraum nicht mehr zum Schaden auch unseres einheimischen Arzneimittelschatzes auslegt und im Endeffekt die Arzneimittelsicherheit in Frage stellt. 


\section{Diplome für nichtärztliche Therapeuten}

Nichtärztliche Therapeuten sind für Patientinnen und Patienten zu einer ersten medizinischen Anlaufstelle geworden. Die Patientensicherheit ist aber nicht gewährleistet, wenn jedermann, auch ohne Ausbildung und Erfahrung, erkrankte Personen behandeln darf. Dies ist aktuell in sechs Kantonen (FR, GE, LU, NE, VD, VS) der Fall. Wie kann ein Therapeut ohne qualifizierte Ausbildung schwere oder ansteckende Krankheitsfälle erkennen, und wie kann bei Epidemien und Pandemien gewährleistet werden, dass Patienten zeitgerecht in ärztliche Obhut gelangen? Im Interesse der Patienten, der Öffentlichkeit und der Ärzteschaft werden einheitliche eidgenössische oder kantonale Diplome verlangt, wie sie das Berufsbildungsgesetz vorsieht. Die Kosten für Ausbildungen, Prüfungen und Diplome werden die Therapeuten tragen. Der Bundesrat (EDI) hat dem zuständigen Bundesamt für Berufsbildung und Technologie (BBT) einen Reglementierungsstopp diktiert, weil er «kein öffentliches Interesse an Diplomen» sieht. Dies ist aus ärztlicher Sicht und im Interesse der «public health» nicht nachvollziehbar.

\section{Parolenfassung FMH}

Die FMH hat in den letzten Jahren die Komplementärmedizin in ihre Gremien integriert, Fähigkeitsausweise [7] erlassen und den Weg zur Zusammenarbeit innerhalb der Ärzteschaft geebnet. Die Öffnung der FMH ist konsequent, betrachtet man den grossen Anteil an Ärzten, die Komplementärmedizin selbst anwenden oder ihr zur Behandlung zuweisen. Die grosse Akzeptanz der Bevölkerung für eine integrative Medizin bedeutet, dass die Zeit zur Beschlussfassung reif ist. Der vorliegende Vorschlag des Parlaments will eine moderate verfassungsmässige Grundlage zur Berücksichtigung der KM im Gesundheitswesen schaffen. Die UNION hat die FMH in den letzten Jahren als verlässlichen Partnerin für komplementärmedizinische Anliegen erlebt und hofft, dass sich die FMH mit der Ja-Parole für eine Stärkung der integrativen Hausarztmedizin ausspricht.

\section{Literatur}

1 BGE 123 V 65 E. 4a vom 20. 2.1997.

2 Schlussbericht Programm Evaluation Komplementärmedizin (PEK) vom 24.4.2006.

3 Handbuch zur Standardisierung der medizinischen und wirtschaftlichen Bewertung medizinischer Leistungen. Bern: BSV; 2000.

4 Die PEK-Studien finden Sie unter: www.bag.admin. ch/themen/krankenversicherung/00263/00264/ 04102/index.html und www.kikom.unibe.ch.

5 www.bag.admin.ch/themen/krankenversicherung/ 00263/00264/04102/index.html (siehe rechte Spalte oben).

6 Statistik der obligatorischen Krankenversicherung 2004. Bern: BAG; 2006.

7 Fähigkeitsausweise bestehen für Homöopathie SVHA, Akupunktur / TCM ASA, Neuraltherapie SANTH und anthroposophische Medizin VAOAS. 